\title{
CORRIGENDUM \\ The abelianization of the congruence IA-automorphism group of a free group
}

\author{
BY TAKAO SATOH \\ (Volume 142 (2007), 239-248) \\ Graduate School of Mathematical Sciences, The University of Tokyo, \\ Komaba, Tokyo, 153-8914, Japan. \\ e-mail: takao@ms.u_tokyo.ac.jp
}

\begin{abstract}
We fill a gap in the construction of the extended Johnson homomorphism, stated in Section 2 of the paper The abelianization of the congruence IA-automorphism group of a free group.
\end{abstract}

(i) Page 5, lines 7-13: For a $\boldsymbol{Z} / d \boldsymbol{Z}$-valued Magnus expansion $\theta$, it is not true if $d$ is even that the image $\operatorname{Im}\left(\tau^{\theta}\right)$ of a crossed homomorphism $\tau^{\theta}$ is $\left(H^{*} \otimes_{z} \Lambda^{2} H\right) \otimes_{z} \boldsymbol{Z} / d \boldsymbol{Z}$ in $\left(H^{*} \otimes_{z} H^{\otimes 2}\right) \otimes_{z} \boldsymbol{Z} / d \boldsymbol{Z}$. If $d$ is odd, it is true. Moreover, if $d$ is even,

$$
\operatorname{Im}\left(\tau^{\theta}\right) \subset\left(\left(H^{*} \otimes_{z} \Lambda^{2} H\right) \otimes_{z} \boldsymbol{Z} / d \boldsymbol{Z}\right) \oplus\left(\frac{d}{2} \cdot(\boldsymbol{Z} / d \boldsymbol{Z})\right)^{\oplus n^{2}} .
$$

Hence for even $d$, we have to modify the construction of the extended Johnson homomorphism, also denoted to by $\tau^{\theta}$,

$$
\tau^{\theta}: I A_{n, d} \longrightarrow\left(H^{*} \otimes_{z} \Lambda^{2} H\right) \otimes_{z} \boldsymbol{Z} / d \boldsymbol{Z}
$$

by composing a natural projection $\operatorname{Im}\left(\tau^{\theta}\right) \rightarrow\left(H^{*} \otimes_{z} \Lambda^{2} H\right) \otimes_{z} \boldsymbol{Z} / d \boldsymbol{Z}$ with a restriction of the crossed homomorphism $\tau^{\theta}$ to $I A_{n, d}$. Although we modify the construction of the extended Johnson homomorphism $\tau^{\theta}$, our result

$$
I A_{n, d}^{\mathrm{ab}} \simeq\left(\left(H^{*} \otimes_{z} \Lambda^{2} H\right) \otimes_{z} \boldsymbol{Z} / d \boldsymbol{Z}\right) \oplus \Gamma(n, d)^{\mathrm{ab}}
$$

remains true for any $d$ as an abelian group. Furthermore we see that this isomorphism is an $\operatorname{SL}(n, \boldsymbol{Z} / d \boldsymbol{Z})$-equivariant for odd $d$, and not for even $d$.

In order to show (1), we use the same notaton as in [1]. Set $R:=\boldsymbol{Z} / d \boldsymbol{Z}$. Define

$$
\Gamma_{2}^{d}:=\left\langle x y x^{-1} y^{-1}, x^{d} \mid x, y \in F_{n}\right\rangle .
$$

It is easy to show that for any $R$-valued Magnus expansion $\theta, \Gamma_{2}^{d}=\theta^{-1}\left(1+\hat{T}_{2}\right)$. Then we have

$$
\theta_{2}\left(\Gamma_{2}^{d}\right)= \begin{cases}\Lambda^{2} H_{R}, & d: \text { odd } \\ \Lambda^{2} H_{R} \oplus\left\langle\frac{d}{2}\left[x_{i}\right]^{\otimes 2} \mid 1 \leqslant i \leqslant n\right\rangle, & d: \text { even, }\end{cases}
$$

Denote by $U$ the right-hand side of (2). It is obvious that $U \subset \theta_{2}\left(\Gamma_{2}^{d}\right)$. To prove $\theta_{2}\left(\Gamma_{2}^{d}\right) \subset U$, 
it suffices to check that the images of the generators of $\Gamma_{2}^{d}$ are in $U$. We easily see that $\theta_{2}\left(x y x^{-1} y^{-1}\right) \in \Lambda^{2} H_{R}$. Furthermore,

$$
\theta\left(x^{d}\right) \equiv 1+\frac{d(d-1)}{2}[x]^{\otimes 2}
$$

modulo $\hat{T}_{3}$. Hence if $d$ is odd, $\theta_{2}\left(x^{d}\right)=0$, and if $d$ is even,

$$
\theta_{2}\left(x^{d}\right)=\frac{d}{2}[x]^{\otimes 2} \in H_{R}^{\otimes 2} .
$$

By writing $[x]=a_{1}\left[x_{1}\right]+\cdots+a_{n}\left[x_{n}\right], a_{i} \in R$, we have

$$
\theta_{2}\left(x^{d}\right)=\frac{d}{2}\left\{\sum_{i=1}^{n} a_{i}^{2}\left[x_{i}\right]^{\otimes 2}+\sum_{i<j} a_{i} a_{j}\left(\left[x_{i}\right] \otimes\left[x_{j}\right]-\left[x_{j}\right] \otimes\left[x_{i}\right]\right)\right\} \in U .
$$

By the argument stated in [1, section 2] we obtain a required crossed homomorphism

$$
\tau^{\theta}: \operatorname{Aut} F_{n} \longrightarrow \operatorname{Hom}_{z}\left(H_{R}, \theta_{2}\left(\Gamma_{2}^{\mathrm{d}}\right)\right)=H_{R}^{*} \otimes_{z} \theta_{2}\left(\Gamma_{2}^{\mathrm{d}}\right),
$$

and (1) is proved.

(ii) Page 6, line 7: $H_{2}\left(I A_{n}, \mathbf{Z}\right)$ and $H_{2}\left(I A_{n, d}, \boldsymbol{Z}\right)$ should read $H_{2}\left(I A_{n, d}, \boldsymbol{Z}\right)$ and $\mathrm{H}_{2}(\Gamma(n, d), \boldsymbol{Z})$ respectively.

(iii) Page 6, line 13: $I A_{n}^{\mathrm{ab}}$ should read $I A_{n, d}^{\mathrm{ab}}$.

Acknowledgements. The author would like to thank Masatoshi Sato and Nariya Kawazumi for discovering and showing me a precise proof for the existence of an extended Johnson homomorphism using the Magnus expansions.

\section{REFERENCE}

[1] N. Kawazumi. Cohomological aspects of Magnus expansions. Preprint, The University of Tokyo, UTMS 2005-18 (2005), arXiv: math. GT/ $0505497 \mathrm{v} 3$. 